\title{
Real-time imaging reveals that lytic polysaccharide monooxygenase promotes cellulase activity by increasing cellulose accessibility
}

Bo Song ${ }^{1 \dagger}$, Bingyao $\mathrm{Li}^{1,2,3 \dagger}$, Xiaoyan Wang ${ }^{1}$, Wei Shen ${ }^{1,3}$, Sungjin Park' ${ }^{1}$, Cynthia Collings ${ }^{1,3}$, Anran Feng ${ }^{1}$, Steve J. Smith ${ }^{4}$, Jonathan D. Walton ${ }^{1,2,3}$ and Shi-You Ding ${ }^{1,3^{*}}$ (i)

\begin{abstract}
Background: The high cost of enzymes is one of the key technical barriers that must be overcome to realize the economical production of biofuels and biomaterials from biomass. Supplementation of enzyme cocktails with lytic polysaccharide monooxygenase (LPMO) can increase the efficiency of these cellulase mixtures for biomass conversion. The previous studies have revealed that LPMOs cleave polysaccharide chains by oxidization of the C1 and/or C4 carbons of the monomeric units. However, how LPMOs enhance enzymatic degradation of lignocellulose is still poorly understood.

Results: In this study, we combined enzymatic assays and real-time imaging using atomic force microscopy (AFM) to study the molecular interactions of an LPMO [TrAA9A, formerly known as TrCel61A) from Trichoderma reesei] and a cellobiohydrolase I (TICel7A from T. longibrachiatum) with bacterial microcrystalline cellulose (BMCC) as a substrate. Cellulose conversion by T/Cel7A alone was enhanced from 46 to 54\% by the addition of TrAA9A. Conversion by a mixture of T/Cel7A, endoglucanase, and $\beta$-glucosidase was increased from 79 to $87 \%$ using pretreated BMCC with TrAA9A for $72 \mathrm{~h}$. AFM imaging demonstrated that individual TrAA9A molecules exhibited intermittent random movement along, across, and penetrating into the ribbon-like microfibril structure of BMCC, which was concomitant with the release of a small amount of oxidized sugars and the splitting of large cellulose ribbons into fibrils with smaller diameters. The dividing effect of the cellulose microfibril occurred more rapidly when TrAA9A and T/Cel7A were added together compared to TrAA9A alone; T/Cel7A alone caused no separation.
\end{abstract}

Conclusions: TrAA9A increases the accessible surface area of BMCC by separating large cellulose ribbons, and thereby enhances cellulose hydrolysis yield. By providing the first direct observation of LPMO action on a cellulosic substrate, this study sheds new light on the mechanisms by which LPMO enhances biomass conversion.

Keywords: LPMO, CBH I, AFM, Lignocellulose, Biomass, Cellulose, Biorefinery

\section{Background}

Non-food plant biomass is a sustainable source of fermentable sugars for the production of biofuels and chemicals [1-3]. Cellulose, the main component of lignocellulosic biomass, forms rigid microfibrils

\footnotetext{
*Correspondence: sding@msu.edu

${ }^{\dagger}$ Bo Song and Bingyao Li contributed equally to this work

1 Department of Plant Biology, Michigan State University, East Lansing, MI 48824, USA

Full list of author information is available at the end of the article
}

composed of well-organized linear $\beta$-1,4-glucan chains. Due to its homogeneity and abundance, cellulose is of major interest in producing monomeric sugars for biorefineries $[2,4,5]$. However, the cellulose chains in a microfibril are strongly held together by hydrogen bond networks and van der Waals forces, so that the majority of these chains are not readily accessible to cellulases. In addition, in plant biomass, microfibrils are organized into closely associated bundles and are embedded in a non-cellulosic matrix of lignin and hemicelluloses, 
which further reduce the efficiency of biomass conversion $[2,6]$.

The most widespread method of cellulose conversion requires three types of cellulases to act synergistically to convert cellulose to glucose: endo- $\beta$-1,4-glucanases (EGs) cleave the internal bonds in the cellulose chain, cellobiohydrolases (CBHs) processively hydrolyze cellulose from the chain ends to produce cellobiose, and $\beta$-glucosidases (BGs) hydrolyze cellobiose to glucose [7]. Lytic polysaccharide monooxygenases (LPMOs) are a recently discovered class of enzymes that stimulate biomass hydrolysis and hence improve the efficiency of biomass conversion [8-11]. Unlike cellulases that cleave glycosidic bonds by hydrolysis [12], LPMOs are copper-dependent enzymes that lyse polysaccharide chains by oxidation at either the $\mathrm{C} 1$ or the $\mathrm{C} 4$ carbon of the glucose unit in the presence of an external electron donor [13-17]. However, the mechanism by which the addition of LPMOs to cellulase mixtures enhances the overall yield of glucose has not been clearly elucidated.

Atomic force microscopy (AFM), especially highspeed AFM, can image with sub-nanometer resolution under aqueous conditions at video rate. AFM has been used to visualize the interactions between enzymes and substrates in real time [18]. AFM has also been used to image the structural changes of cellulose after treatment by LPMO showing disintegrating and fibrillation of cellulose microfibrils [19]. However, how the interaction of LPMO with the cellulose surface causes these changes is still not well understood. In this study, we use high-speed AFM to monitor in situ interactions of an LPMO (TrAA9A, formerly known as $\operatorname{TrCel61\mathrm {A})}$ and a CBH I ( $T l C e l 7 \mathrm{~A})$, alone and together, with bacterial microcrystalline cellulose (BMCC). Our goal was to visualize in real time the molecular motion of LPMO and its effects on the structure of the cellulose surface, to deepen our understanding of LPMO's role in cellulose hydrolysis.

\section{Results and discussion}

$\mathrm{CBH} I$ is a major cellulase that degrades crystalline cellulose [7, 20]. LPMO has been reported to enhance $\mathrm{CBH}$ I hydrolysis of pretreated biomass $[8,11]$. Under our experimental conditions, a mixture of TrAA9A and $T l$ Cel7A converted $8 \%$ more cellulose ( $46 \%$ compared to $54 \%$ of the theoretical maximum yield) than $T l \mathrm{Cel} 7 \mathrm{~A}$ alone (Fig. 1). This result is consistent with the previous work showing that hydrolysis of cellulose in pretreated corn stover increased approximately $6 \%$ by adding a Thielavia terrestris LPMO to $T$. reesei cellulase mixtures [8]. Therefore, we assume that the TrAA9A used in this study exhibits similar overall activity in enhancing cellulose hydrolysis by $T l \mathrm{Cel} 7 \mathrm{~A}$.

LPMO has been shown to break cellulose chains by oxidation at the $\mathrm{C} 1$ and/or $\mathrm{C} 4$ position(s) [4, 21, 22]. To further confirm the specific activity of $\operatorname{Tr}$ AA9A, we

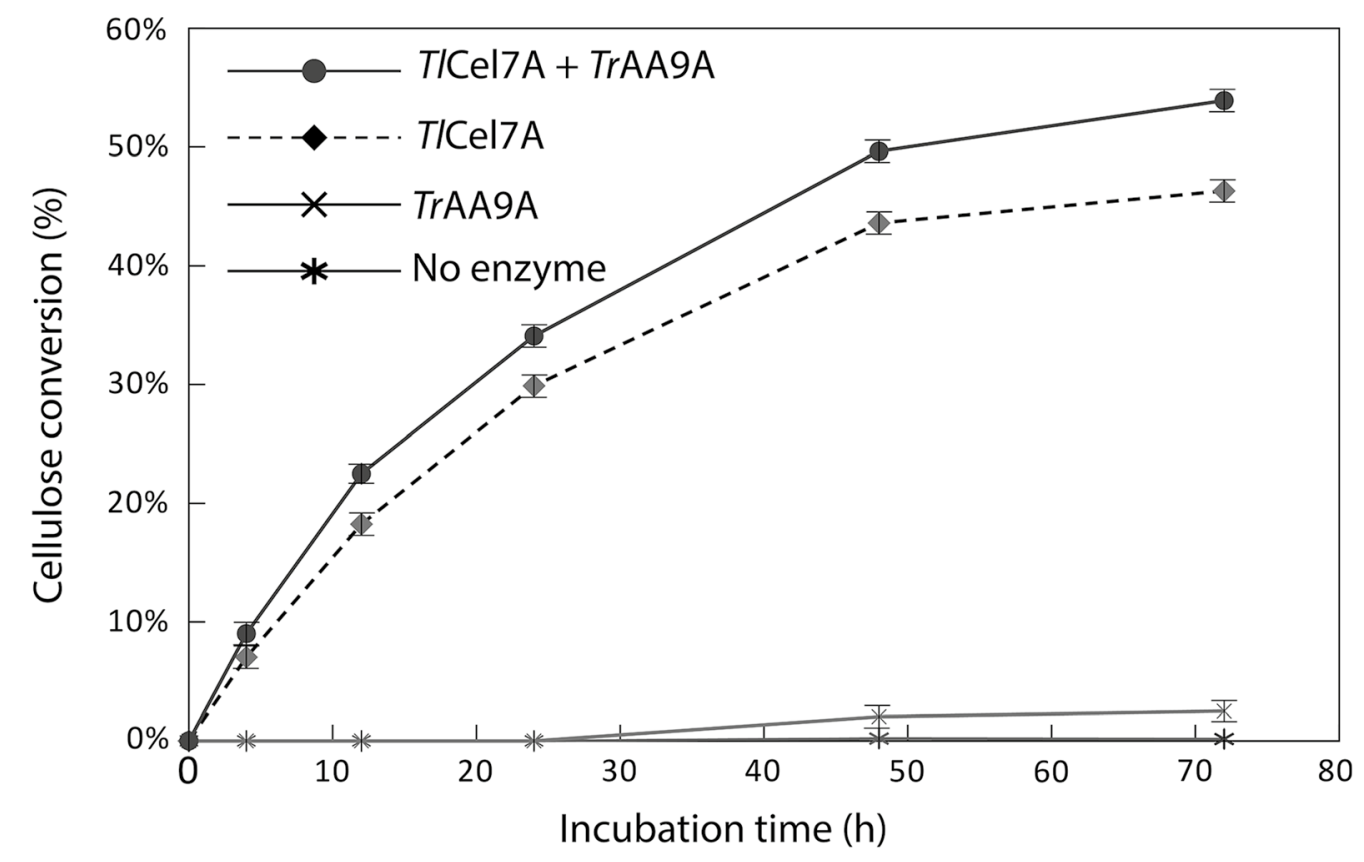

Fig. 1 Cellulose conversion by TrAA9A and T/Cel7A. BMCC ( $2 \mathrm{mg} / \mathrm{mL})$ was treated with T/Cel7A ( $34 \mu \mathrm{g} / \mathrm{mL}$ ) alone or together with TrAA9A (6 $\mu \mathrm{g} /$ $\mathrm{mL}$ ) in 50-mM sodium acetate buffer ( $\mathrm{pH} 4.8)$ containing 1-mM L-ascorbic. The reaction was carried out at $50^{\circ} \mathrm{C}$ with agitation at $150 \mathrm{rpm}$. The error bars represent the standard deviation of the triplicates 
used mass spectrometry (MS) to analyze the soluble products after $72 \mathrm{~h}$ incubation of TrAA9A with BMCC, and detected small amounts of oxidized forms of cellobiose and cellotriose (Additional file 1: Figure S1), consistent with the previous reports [23].

To visualize TrAA9A interaction with BMCC under AFM, a small piece of BMCC was transferred onto a glass slide pre-coated with poly-lysine. As the negative control, BMCC was imaged under the same buffer used for the enzyme reactions. We observed primarily large ribbon-like cellulose structures of 30-150-nm width, with a relatively smooth surface. About $10 \mathrm{~min}$ after adding TrAA9A, we observed particles of $6-10 \mathrm{~nm}$ in diameter accumulating on the surface of the BMCC ribbon (Fig. 2). TrAA9A was predicted to be $\sim 6 \mathrm{~nm}$ in diameter based on the structure of TaGH61 [14,
24], and we, therefore, considered these particles to be bound TrAA9A molecules.

Continuous imaging revealed that $\operatorname{TrAA9A}$ molecules initially bound randomly to the BMCC surface, and individual TrAA9A molecules exhibited a "stop-and-go" behavior. Interestingly, the enzyme molecules stayed in approximately the same position for a much longer time than they spent moving on the cellulose. TrAA9A moved at $0.25 \pm 0.13 \mathrm{~nm} / \mathrm{s}(n=40)$ in three patterns: in both directions along one cellulose ribbon, across one ribbon, or moving from one ribbon to another (Fig. 2a-c, respectively; details in Additional file 2: Video S1, Additional file 3: Video S2, Additional file 4: Video S3). The observed speed of TrAA9A was one order of magnitude slower than previously observed for $\mathrm{CBHI}$ moving on Valonia microcrystalline cellulose ( $\operatorname{Tr} \mathrm{Cel} 7 \mathrm{~A})$ [25]. This

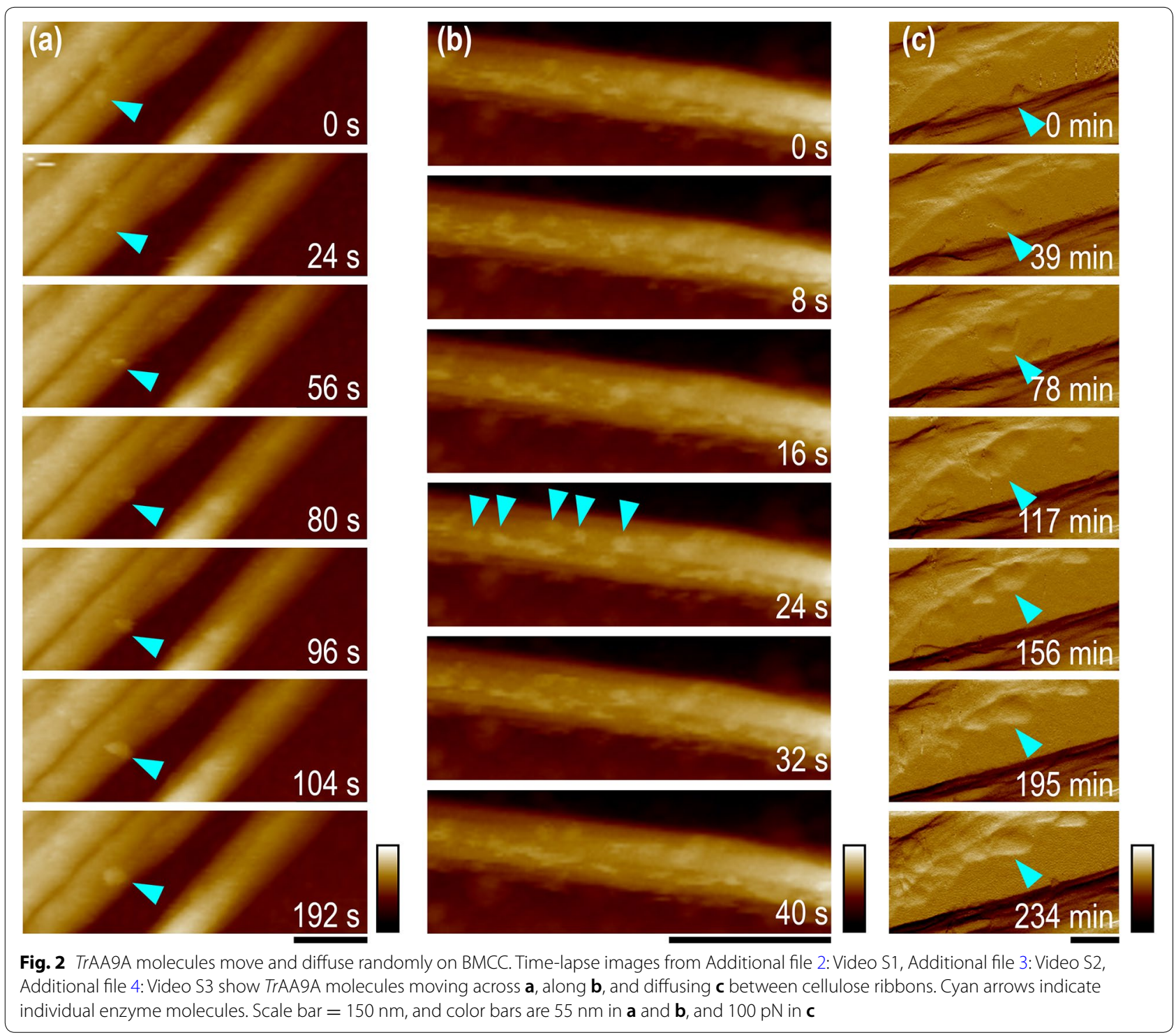


might be related to the slow rate of oxidization catalyzed by $\operatorname{TrAA9A}$ [16]. Interestingly, when the imaging period was extended to $\sim 7 \mathrm{~h}$, some $\operatorname{TrAA9A}$ molecules seemed to form into groups that moved together towards a particular cellulose ribbon where eventually many molecules accumulated (Fig. 2c; Additional file 4: Video S3).

Previously, AFM imaging revealed that $\operatorname{Tr}$ Cel7A binds and reacts on the hydrophobic face of crystalline cellulose [26] and moves processively from the reducing end of the glucan chain to the non-reducing end [25]. In contrast, TrAA9A moves in both directions along a ribbon and also across ribbons. The different binding and movement patterns of TrAA9 and TlCel7A observed in this study can be explained by the differences in their molecular structures. The binding face of LPMO is flat [14, 24], whereas CBHs like $T l \mathrm{Cel}$ A adopt a tunnel-shape active site that can hold several glucose residues of the cellulose chain and thereby constrain movement in a linear fashion [21, 22, 27, 28].

By monitoring the height change in the region where individual TrAA9A enzymes crossed, we observed the unexpected phenomenon of enzyme molecules penetrating inside the ribbon (Fig. 3, Additional file 5: Figure S2, and Additional file 6: Video S4). After the addition of $\operatorname{TrAA9A}$, there was an initial increase in height due to the bound enzyme. Subsequently, TrAA9A molecules penetrated inside the cellulose ribbon and were hardly visible after $27 \mathrm{~min}$. The enzyme gradually came back to the surface after $96 \mathrm{~min}$, moved along the cellulose surface, and diffused away after $210 \mathrm{~min}$. As TrAA9A moved, grooveshape features appeared on the cellulose microfibril surface and the existing grooves deepened and widened. As a result, more obvious edges appeared on the cellulose surface after 2-h incubation with TrAA9A (Additional file 7: Figure S3, and Additional file 8: Video S5).

The width of cellulose microfibrils was measured during incubation with TrAA9A (Fig. 4). After 4-h treatment, the average width of the cellulose ribbons slightly decreased from $101.8 \pm 14.9 \mathrm{~nm}(n=20)$ to $89.8 \pm 16.7 \mathrm{~nm}(n=16)$, but the surface roughness increased greatly, with obvious grooves and edges observed on the surface (Fig. 4b) compared to a relatively smooth surface before treatment (Fig. 4a). After 24-h incubation, we observed splitting of the ribbon-like cellulose microfibrils along its long axis into smaller microfibrils of $52.9 \pm 11.7 \mathrm{~nm}(n=24)$ in width, which was approximately $52 \%$ of their original ribbon width. Similar structural change was also observed recently [19] using bleached softwood Kraft pulp as the substrate treated with LPMO. We postulate that the ability of TrAA9A to penetrate inside the cellulose ribbon leads to the dividing of a large cellulose ribbon into multiple smaller microfibrils. Considering that a BMCC ribbon is composed of multiple cellulose elementary fibrils (CEF) that can be as small as $2-4 \mathrm{~nm}[29,30]$, it is possible that TrAA9A oxidation disrupts the glucan chains between these CEFs. We further studied the overall $\mathrm{BMCC}$ structure changes by incubating $T l C e l 7 \mathrm{~A}$ with and without $\operatorname{TrAA} 9 \mathrm{~A}$. After 72-h incubation with both enzymes, BMCC was broken into small pieces. In contrast, treatment with $T l C e l 7 \mathrm{~A}$ alone resulted in softening of BMCC floating on the top of the reaction buffer. There was no significant structural change of BMCC viewed by eye after TrAA9A treatment alone (Additional file 9: Figure S4). AFM was used to further visualize the structural changes of individual BMCC ribbon after treatment of these two enzymes at the nanometer scale (Fig. 5). For the first $20 \mathrm{~min}$, the average height and width of the cellulose ribbon slightly increased, which was likely due to the binding of TrAA9A and $T l C e l 7 \mathrm{~A}$ onto the cellulose surface. After 7-h incubation, the height of the BMCC ribbon decreased, while the width increased. A significant decrease in height and increase in width was observed from 106 to $149 \mathrm{~min}$, which was concomitant with the disintegration of the large cellulose ribbon into small microfibrils. Between 213 and $255 \mathrm{~min}$, the cellulose ribbon completely separated into small microfibrils of $\sim 4 \mathrm{~nm}$ in diameter, as the ribbon height continued to decrease and the width to increase (Fig. 5a, details in Additional file 10: Video S6). The diameter of the small microfibril $(\sim 4 \mathrm{~nm})$ indicated that they could contain an individual or a bundle of at most a few fundamental CEFs [29, 30].

After $4 \mathrm{~h}$ of incubation, the resulting cellulose microfibrils were significantly thinner when treated by TrAA9A together with $T l C e l 7 \mathrm{~A}$ (Fig. 5a) than when treated by TrAA9A alone (Fig. 4). Interestingly, cellulose hydrolysis by the cellulase mixture composed of $T l \mathrm{Cel} 7 \mathrm{~A}, \mathrm{EG}$, and BG increased from 79 to $87 \%$ when using TrAA9A-pretreated BMCC, compared with untreated BMCC (Additional file 11: Figure S5). This enhancement is similar to the results for $\operatorname{Tr} \mathrm{AA} 9 \mathrm{~A}$ and $T l \mathrm{Cel} 7 \mathrm{~A}$ added together to untreated BMCC, suggesting that the synergy of TrAA9A and cellulases during cellulose hydrolysis may not be attributed to the direct molecular interaction between these enzymes.

\section{Conclusions}

We used high-speed AFM imaging to visualize in realtime the enzymatic hydrolysis of cellulose by cellulases and an LPMO, $\operatorname{TrAA9A}$, in this study. Our results are consistent with the previous studies, but further show that cellulases, i.e., $\mathrm{CBHs}$ and EGs, are responsible for the majority of cellulose hydrolysis, and that LPMOs promote their efficiency. We observe that LPMO reacts specifically to disintegrate large cellulose ribbons into small microfibrils with little oxidized sugar products, whereas CBHI 

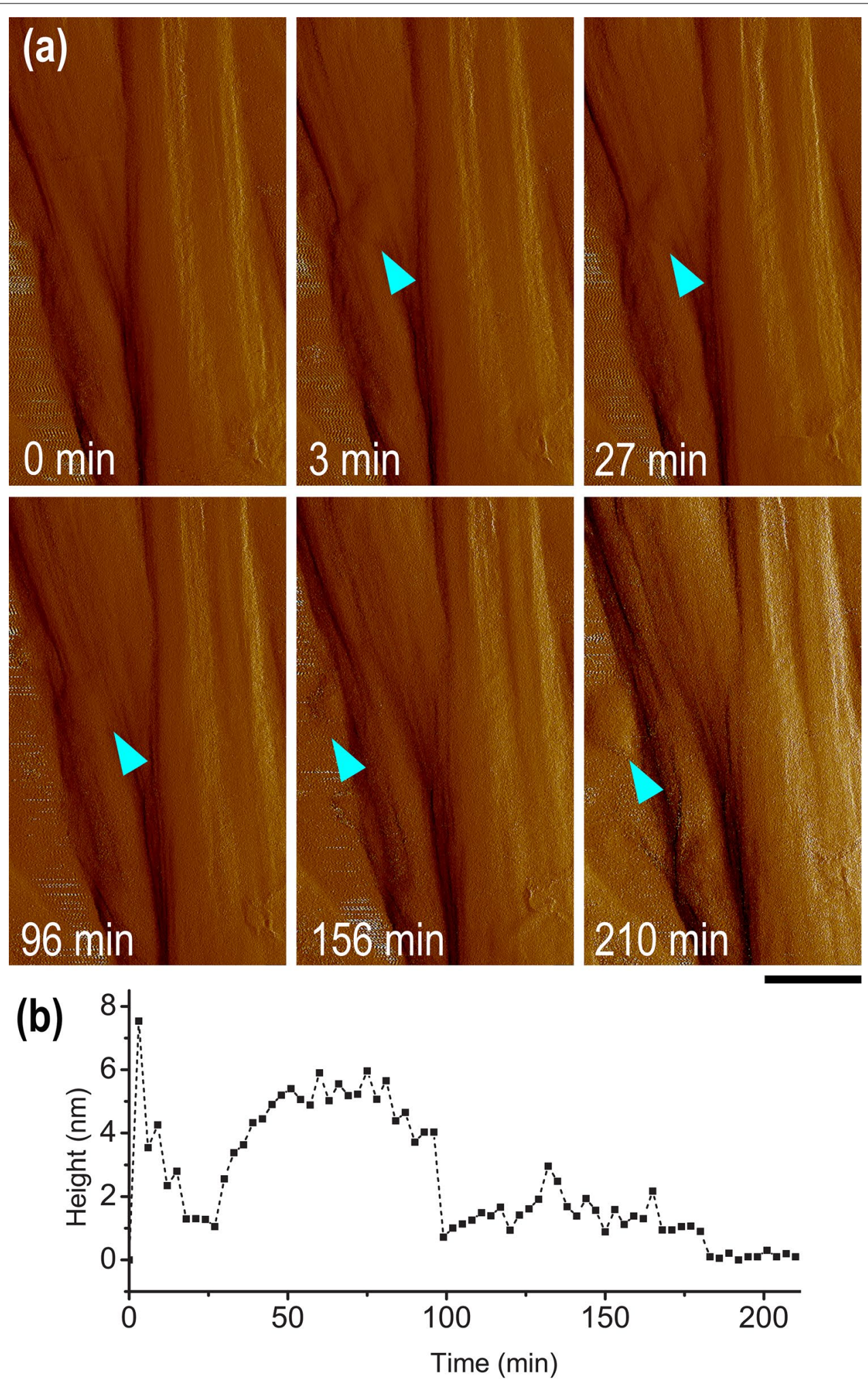

Fig. 3 TrAA9A penetrating and moving inside a BMCC ribbon. a Time-lapse AFM peak force error images showing TrAA9A (indicated by the cyan arrows) moving in and out of the surface of a cellulose ribbon (See Additional file 5: Figure S2 and Additional file 6: Video S4 for more information). $\mathbf{b}$ Relative height measured across a TrAA9A molecule during 210 min incubation. Scale bar is $100 \mathrm{~nm}$ and color bar is $1.1 \mathrm{nN}$ 

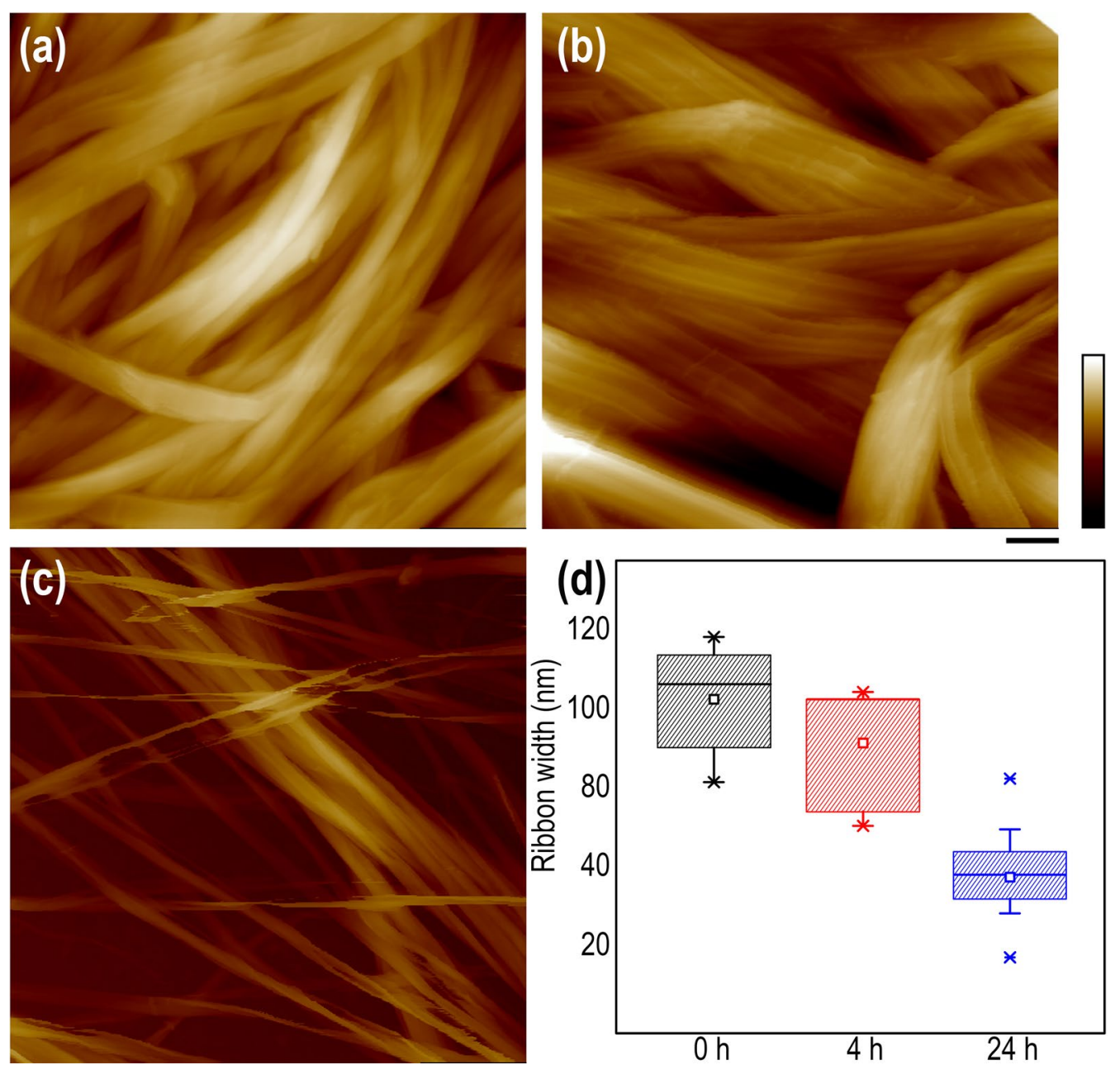

Fig. 4 Disassembly of BMCC ribbon into smaller fibrils by TrAA9A. Atomic force micrographs of BMCC before $\mathbf{a}$, after 4-h $\mathbf{b}$ and after 24-h c incubation with TrAA9A. $\mathbf{d}$ Width (and standard deviation) of BMCC microfibrils before and after TrAA9A treatment. Scale bar is $150 \mathrm{~nm}$ and color bar is $200 \mathrm{~nm}$

has been considered to be a "peel-off" process [26, 31]; we hypothesize that LPMO and CBHI may attack different structures of cellulose. BMCC ribbons are considered to contain amorphous cellulose and CEFs that are composed of well-organized cellulose glucan chains [29, 30]. It is likely that LPMO attacks only the amorphous proportion of cellulose and increases the surface accessibility of CEFs for cellulase enzymes. If this is true, LPMO would not enhance cellulase activity when cellulose accessibility is already at a maximum. Indeed, it has been previously demonstrated that the degree of LPMO enhancement is negatively correlated with cellulose accessibility [9]. Similarly, LPMO has been found to increase the degradation of insoluble xylan [32], one of the major matrix polysaccharides in the plant cell wall, suggesting that LMPO can oxidize sugars in different amorphous polysaccharides, thus exposing the well-organized cellulose microfibrils to be hydrolyzed by cellulases.

\section{Methods}

General chemicals

All chemicals and reagents, unless specifically noted, were purchased from Sigma-Aldrich (St. Louis, MO).

\section{Bacterial strain and medium}

Gluconacetobacter xylinus (also known as Komagataeibacter xylinus) strain ATCC 53524 from the AmericanType Culture Collection (Manassas, VA) was maintained on Hestrin and Schramm (HS) medium containing 1.5\% agar. HS medium contains $20-\mathrm{g} / \mathrm{L}$ glucose, 5 -g/L peptone, $5-\mathrm{g} / \mathrm{L}$ yeast extract, $2.7-\mathrm{g} / \mathrm{L} \mathrm{Na}_{2} \mathrm{HPO}_{4}$, and $1.15-\mathrm{g} / \mathrm{L}$ 

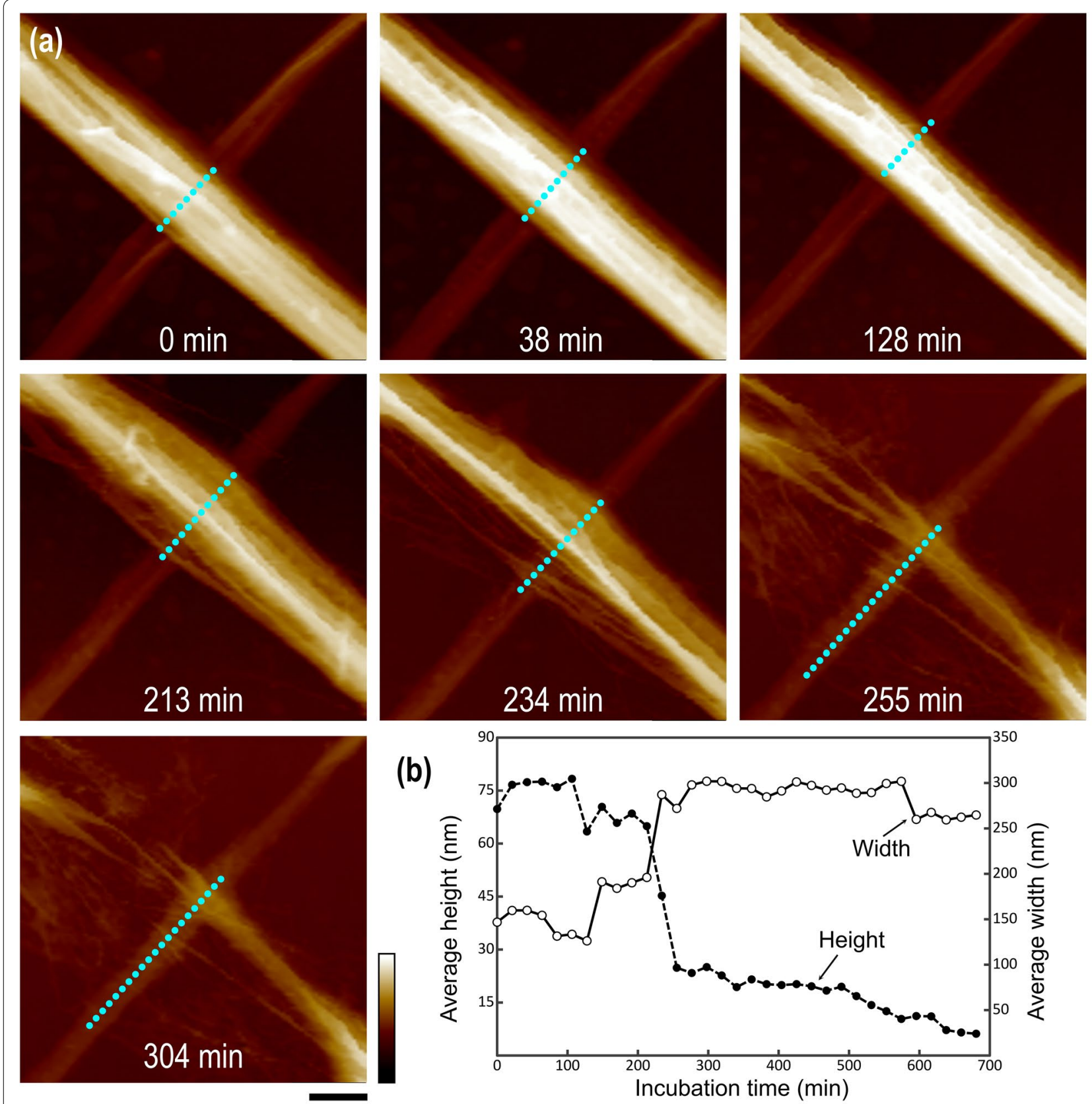

(c)
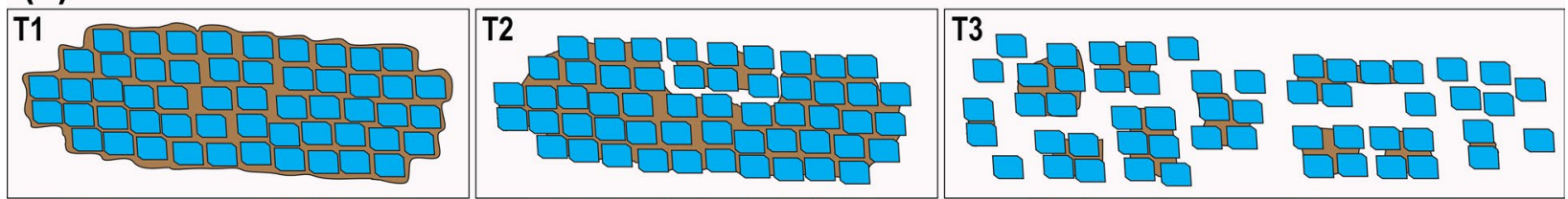

Fig. 5 Synergism between TrAA9A and T/Cel7A during hydrolysis of BMCC. a Time-lapse images from Additional file 10: Video S6 showing changes in the appearance of the cellulose ribbon during incubation with TrAA9A and T/Cel7A. b Height and width measurements. Cyan dash lines in $\mathbf{a}$ indicate the overall width change of a BMCC ribbon during enzymatic treatment. Scale bar is $50 \mathrm{~nm}$, and color bar is $100 \mathrm{~nm}$ 
citric acid monohydrate. The $\mathrm{pH}$ was adjusted to 6.0 with $\mathrm{NaOH}$ or $\mathrm{HCl}$.

\section{Enzymes}

TlCel7A from Trichoderma longibrachiatum (Cata$\log$ No. E-CBHI) was purchased from Megazyme, Ltd., Bray, Ireland. TrAA9A (GenBank CAA71999), endo- $\beta$ 1,4-glucanase (EG; AAA34212) and $\beta$-glucosidase (BG; AAA18473) from $T$. reesei were expressed in Pichia pastoris as previously described [33]. Pronase E (Catalog No. P2714) was purchased from Sigma-Aldrich (St. Louis, MO).

\section{Production of BMCC}

The bacterial microcrystalline cellulose (BMCC) was produced based on the previous study with modifications $[34,35]$. HS medium $(50 \mathrm{~mL})$ in a $250-\mathrm{mL}$ flask was inoculated with G. xylinus colonies and incubated at $30^{\circ} \mathrm{C}$ for 1-3 days in static until cellulose pellicle was visible. The culture was vigorously shaken for $30 \mathrm{~min}$ to release active cells embedded in the pellicle. Then, 450-mL fresh HS medium in a plastic tray $(18 \times 6 \times 2.5$ in. $)$ was inoculated with the culture broth and incubated in stationary for another 3-5 days until the cellulose pellicle was grown on the entire medium surface.

The cellulose pellicle was collected by filtering through 2-layer Miracloth. Then, the pellicle was washed repeatedly with distilled deionized water $\left(\mathrm{ddH}_{2} \mathrm{O}\right)$ to rinse off the medium as much as possible. Washed cellulose was boiled in $1 \% \mathrm{NaOH}$ with stirring for $30 \mathrm{~min}$ to remove remaining bacteria. Finally, alkali-treated cellulose was washed several times with $\mathrm{dd}_{2} \mathrm{O}$ until the water $\mathrm{pH}$ reached 7.0, freeze-dried, and cut to 1-cm strip for enzymatic degradation. Native BMCC without alkali treatment was stored in $0.02 \%$ sodium azide at $4{ }^{\circ} \mathrm{C}$ for AFM imaging.

\section{Preparation of BMCC samples}

The BMCC sample was prepared by a hand-cutting of fresh and never dried BMCC film. Only thin layers with approximated $10-50-\mu \mathrm{m}$ thickness were used in the experiment. A bright field microscopy was used to select a thinner sample with relative uniform surface. After washing by deionized water several times and placed on a poly-lysine-coated glass slide (Thermo Fisher Scientific Inc, Waltham, MA, USA) with deionized water. The samples were kept in the water for all of the imaging and measurement process.

\section{AFM operation}

All the AFM experiments were conducted at room temperature on a Dimension AFM with Nanoscope controller V (Fastscan, Bruker Nano, Santa Barbara, CA. USA) with an acoustic and vibration isolation system. Probes we used were SCANASYST-FLUID+ (Bruker, Camarillo, CA USA) for imaging under fluid. The AFM operation software (Nanoscope V9.1) was used to control the scan size, setpoint, and gain. Before AFM imaging, the scanner has been carefully calibrated using calibration kit (Bruker, Camarillo, CA, USA) to make sure that all the measurements are very close to their actual value. To dynamically capture the movement of enzyme molecules, the scan rate was normally set as $10-20 \mathrm{~Hz}$ for the continuous observation with low resolution $(256 \times 128$ pixels or $128 \times 64$ pixels). However, when we conducted the imaging on single TrAA9A penetration experiment, we lowered the scan rate to $2 \mathrm{~Hz}$ to obtain images with better resolution. For static observations, the scan rate was $1 \mathrm{~Hz}$ with the resolution of $1024 \times 1024$ pixels.

\section{AFM measurement and image processing}

All off-line data analysis was based on the Nanoscope Analysis v1.8 software (Bruker Nano, Santa Barbara, CA. USA). The height and peak force error images were analyzed using plane fit filter at one order for images presented in all figures. The color bar was manually modified according to the best presence of each image. The images used for height and width measurement were raw data without any processing and all width and height measurements were conducted with the "section" function in the Nanoscope Analysis software.

\section{Preparation of TrAA9A-treated BMCC}

$2-\mathrm{mg} / \mathrm{mL}$ BMCC was incubated with $100-\mu \mathrm{g} / \mathrm{mL}$ $\operatorname{Tr}$ AA9A, 1-mM $\mathrm{L}_{\mathrm{L}}$-ascorbic acid, and $0.02 \%$ sodium azide for $72 \mathrm{~h}$ in $50-\mathrm{mM} \mathrm{pH} 4.8$ sodium acetate buffer. All reactions in triplicate were conducted at $150 \mathrm{rpm}, 50{ }^{\circ} \mathrm{C}$ in shaking incubator. After 72-h incubation, $\mathrm{BMCC}$ residue was recovered by filtering through two layers of Miracloth (Calbiochem, San Diego, CA). Filtrates from all reactions were saved for $\operatorname{TrAA} 9 \mathrm{~A}$ product analysis. Then, TrAA9A remained on cellulose residue was removed according to the previous study with modifications [36]. The residue was first washed several times with $\mathrm{ddH}_{2} \mathrm{O}$. Then, it was incubated with $10-\mathrm{mg} / \mathrm{mL}$ Pronase $\mathrm{E}$ in $50-\mathrm{mM} \mathrm{pH} 7.5$ Tris buffer overnight at $37^{\circ} \mathrm{C}, 100 \mathrm{rpm}$ for complete proteolysis of remaining TrAA9A. Next, BMCC residue was collected by filtering through two layers of Miracloth, washed with $\mathrm{dd}_{2} \mathrm{O}, 1 \mathrm{M} \mathrm{NaCl}$, and $\mathrm{ddd}_{2} \mathrm{O}$ again to remove Pronase $\mathrm{E}$. Finally, the $\mathrm{BMCC}$ residue was freeze-dried and stored at $4{ }^{\circ} \mathrm{C}$.

\section{TrAA9A product analysis by LC-MS}

Filtrate obtained after 72 -h reaction with $\operatorname{TrAA9A}$ was centrifuged in $70 \%$ ethanol for $20 \mathrm{~min}$ at $4{ }^{\circ} \mathrm{C}$ to 
precipitate TrAA9A and supernatant was collected. Filtrate of BMCC incubated under the same condition without $\operatorname{Tr}$ AA9A was also prepared in the same way for mass spectrometry analysis. Samples were analyzed on a Waters Xevo G2-XS Q-TOF system coupled to a Waters I-Class UPLC system. Carbohydrates were separated by an ACQUITY UPLC BEH Amide column $(2.1 \times 100 \mathrm{~mm}$, $1.7 \mu \mathrm{m}$ ) maintained at $40^{\circ} \mathrm{C}$, with the injection volume at $10 \mu \mathrm{L}$. Solvent A was $10-\mathrm{mM}$ ammonium formate, and solvent B was $100 \%$ acetonitrile. The solutes were eluted at $0.2 \mathrm{~mL} / \mathrm{min}$ starting at $95 \% \mathrm{~B}$, followed by a linear gradient to $35 \% \mathrm{~B}$ over $14 \mathrm{~min}$. The proportion of solvent $\mathrm{B}$ was maintained at $35 \%$ for 2 min and then increased back to $95 \%$ and kept for $4 \mathrm{~min}$ for re-equilibration.

The operation condition for mass spectrometer was capillary voltage of $3.0 \mathrm{kV}$, sample cone voltage of $80 \mathrm{~V}$, source temperature of $100{ }^{\circ} \mathrm{C}$, desolvation temperature of $350{ }^{\circ} \mathrm{C}$, and desolvation gas flow of $600 \mathrm{~L} / \mathrm{h}$. Mass spectra were acquired in positive ion mode across the 50-2000$\mathrm{m} / \mathrm{z}$ range. Data processing was performed using the MassLynx software (version 4.1, Waters).

\section{Enzymatic degradation of BMCC}

To test the synergism between $\operatorname{TrAA9A}$ and $T l$ Cel7A on cellulose degradation, $2-\mathrm{mg} / \mathrm{mL}$ BMCC was incubated with $34-\mu \mathrm{g} / \mathrm{mL}$ TlCel7A and $6-\mu \mathrm{g} / \mathrm{mL}$ TrAA9A, alone or together, in $10-\mathrm{mL}$ reactions containing $1-\mathrm{mM}$

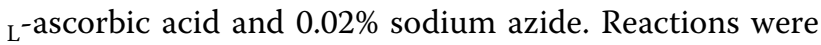
conducted in triplicate in 50-mM sodium acetate, $\mathrm{pH} 4.8$, for $72 \mathrm{~h}$ at $150 \mathrm{rpm}, 50^{\circ} \mathrm{C}$. To detect cellulose conversion rate, $100-\mu \mathrm{L}$ supernatant was incubated with $40-\mu \mathrm{g} / \mathrm{mL}$ $\beta$-glucosidase in $50-\mathrm{mM} \mathrm{pH} 4.8$ sodium acetate buffer for $30 \mathrm{~min}$ at $50{ }^{\circ} \mathrm{C}$. Free glucose was measured by enzymelinked colorimetry as described [33].

To test the digestibility of TrAA9A-treated cellulose, TrAA9A-treated BMCC was compared against untreated BMCC for enzymatic hydrolysis at $\mathrm{pH} 4.8$. For all reactions, cellulose loading was $2 \mathrm{mg} / \mathrm{mL}$ and cellulase mixture loading $100 \mu \mathrm{g} / \mathrm{mL}$, which contained $T l$ Cel7A, EG, and BG with 6:3:1 ratio. Each reaction in triplicate was carried at $150 \mathrm{rpm}, 50{ }^{\circ} \mathrm{C}$ in $0.02 \%$ sodium azide. Glucose yield was measured during $72-\mathrm{h}$ incubation.

\section{Additional files}

Additional file 1: Figure S1. ESI-MS analysis of oxidized cellobiose and cellotriose released from BMCC by TrAA9A. BMCC $(2 \mathrm{mg} / \mathrm{mL})$ was incubated with $100 \mu \mathrm{g} / \mathrm{mL}$ TrAA9A and $1 \mathrm{mM} \mathrm{L}$-ascorbic acid in $50 \mathrm{mM}$ sodium acetate, $\mathrm{pH} 4.8$, for $72 \mathrm{~h}$ at $50^{\circ} \mathrm{C}$ and $100 \mathrm{rpm}$. The reactions were conducted in triplicate. As the negative control, BMCC alone was incubated for $72 \mathrm{~h}$ under the same condition. (a) Small amount of cellobiose was detected in the negative control. (b), (c), and (d) Cellobiose (DP2), cellotriose (DP3), and two oxidation products derived from DP2 and
DP3 were detected in all replicates of TrAA9A-containing samples. Lytic polysaccharide monooxygenase (LPMO) degrades cellulose to cellooligosaccharides and oxidized cello-oligomers. Three types of LPMOs were described based on their oxidation products. Type 1 enzymes oxidize the $\mathrm{C} 1$ carbon of the glucose unit and produce aldonolactone and its hydrated product aldonic acid [15, 16, 37, 38]. Type 2 enzymes oxidize C4 carbon and generate gem-diol intermediate and 4-ketoaldose [10, 23, 39]. Type 3 oxidizes both $\mathrm{C} 1$ and $\mathrm{C} 4$ carbons and could generate $\mathrm{C} 1$ oxidation product, C4 oxidation product, and C1-C4 double-oxidized products [37, $39,40-43]$. C1 or C 4 oxidation alone can lead to the breakage of cellulose chain. Products of TrAA9A were analyzed by mass spectrometry. After 72 h incubation, BMCC alone produced a small amount of cellobiose (DP2) but no other cellodextrin or oxidized sugar (Fig. S1a). In contrast, TrAA9A generated cellobiose (DP2), cellotriose (DP3), and two types of oxidation products derived from DP2 and DP3 after $72 \mathrm{~h}$ reaction (Fig. S1b, c, d). Cellodextrin and oxidized sugar products were all associated with $\mathrm{Na}^{+}$ in mass spectrum probably because the reactions were conducted in sodium acetate buffer [44]. Although there was a small amount of cellobiose in the negative control, its relative intensity is much lower than that from TrAA9A-containing reactions. Therefore, cellobiose is probably one of the products of TrAA9A. In contrast to many studies, cello-oligomer and oxidized cello-oligomer of higher degree of polymerization (DP) were not detected after $72 \mathrm{~h}$ TrAA9A reaction. One possible explanation is that TrAA9A might also be active on cello-oligosaccharide [23] and that cellodextrins of higher DP were degraded after $72 \mathrm{~h}$ reaction. Due to the identical masses between gem-diol and aldonic acid (DPx + $16 \mathrm{amu}$, DPx represents cellodextrin), and between ketoaldose and aldonolactone (DPx - 2 amu), MS analysis alone cannot determine the type of TrAA9A activity [23].

Additional file 2: Video S1. AFM (Height) of TrAA9A molecules moved and diffused across on BMCC cellulose ribbons for approximately $10 \mathrm{~min}$. The images were recorded at $128 \times 64$ pixels, and $17-\mathrm{Hz}$ scan rate. The height scale was fixed at $100 \mathrm{~nm}$. The movie is played back at $\sim 650$ times of actual speed.

Additional file 3: Video S2. AFM (Height) of TrAA9A molecules moved and diffused along on BMCC cellulose ribbons for approximately $10 \mathrm{~min}$. The images were recorded at $128 \times 42$ pixels, and $17-\mathrm{Hz}$ scan rate. The height scale was fixed at $35.5 \mathrm{~nm}$. The movie is played back at $~ 650$ times of actual speed.

Additional file 4: Video S3. AFM (Peak force error) of TrAA9A molecules moved and jumped between BMCC cellulose ribbons for approximately 6 hours. The images were recorded at $256 \times 256$ pixels, and 1.5- Hz scan rate. The height scale was fixed at $1.3 \mathrm{nN}$. The movie is played back at $~ 1800$ times of actual speed.

Additional file 5: Figure S2. TrAA9A penetrating and moving inside BMCC ribbon. Time-lapse images showing TrAA9A (indicated by the cyan arrows) moving in and out of the surface of a cellulose ribbon during 210 min incubation. Scale bar is $100 \mathrm{~nm}$ and color bar $75 \mathrm{~nm}$.

Additional file 6: Video S4. AFM (Peak force error) of TrAA9A molecules penetrated and moved inside BMCC cellulose ribbons for approximately 3 hours. The images were recorded at $256 \times 256$ pixels, and $1.5-\mathrm{Hz}$ scan rate. The height scale was fixed at $0.8 \mathrm{nN}$. The movie is played back at 1800 times of actual speed.

Additional file 7: Figure S3. Changes in morphology of BMCC ribbon when incubated with TrAA9A (indicated by cyan arrows) during $2 \mathrm{~h}$ continuous AFM observation. Pictures were taken from Video S5. Scale bar is $50 \mathrm{~nm}$. Color bar is $50 \mathrm{~nm}$ and $830 \mathrm{pN}$ in height (left) and peak force error (right) channels, respectively.

Additional file 8: Video S5. AFM (Height) of TrAA9A molecules moved and caused morphology change of BMCC cellulose ribbon for approximately $2 \mathrm{~h}$. The images were recorded at $128 \times 64$ pixels, and $17-\mathrm{Hz}$ scan rate. The height scale was fixed at $50 \mathrm{~nm}$. The movie is played back at 650 times of actual speed.

Additional file 9: Figure S4. Morphology change of BMCC after $72 \mathrm{~h}$ incubation with or without enzymes. Reaction conditions were the same 
as Fig. 1. BMCC was cut to $1-\mathrm{cm}$ strip for enzymatic digestion. Cellulose loading was $2 \mathrm{mg} / \mathrm{mL}$, T/Cel7A $34 \mu \mathrm{g} / \mathrm{mL}$, and TrAA9A $6 \mu \mathrm{g} / \mathrm{mL}$. Reactions were conducted in triplicates in $50 \mathrm{mM}$, pH 4.8, sodium acetate buffer at $150 \mathrm{rpm}$ and $50^{\circ} \mathrm{C}$. No obvious morphological change of cellulose strip after TrAA9A treatment was observed. In contrast, after $72 \mathrm{~h}$ incubation with T/Cel7A, the length of the strip shortened to approximately half of its original length with almost $50 \%$ cellulose degradation (Fig. 1). Surprisingly, after $72 \mathrm{~h}$ incubation with both TrAA9A and T/Cel7A, the cellulose gel was broken into small insoluble particles (mostly floating on surface in the picture).

Additional file 10: Video S6. AFM (Height) of morphological changes of cellulose ribbon during incubation with TrAA9A and T/Cel7A for approximately 6 hours. The images were recorded at $256 \times 256$ pixels, and 10- $\mathrm{Hz}$ scan rate. The height scale was fixed at $100 \mathrm{~nm}$. The movie is played back at 1000 times of actual speed.

Additional file 11: Figure S5. Comparison of cellulase hydrolysis of untreated and TrAA9A-treated BMCC. Cellulose loading was $2 \mathrm{mg} / \mathrm{mL}$ and total cellulase loading was $100 \mathrm{\mu g} / \mathrm{mL}$ with T/Cel7A: EG: BG ratio of 6:3:1. The reactions in triplicate were conducted in $50 \mathrm{mM}$, $\mathrm{pH}$ 4.8, sodium acetate buffer at $150 \mathrm{rpm}, 50^{\circ} \mathrm{C}$ for $72 \mathrm{~h}$.

\section{Abbreviations}

LPMO: Iytic polysaccharide monooxygenase; CBH: cellobiohydrolase; TrAA9A: a lytic polysaccharide monooxygenase from Trichoderma reesei; T/Cel7A: a cellobiohydrolase I from Trichoderma longibrachiatum; BMCC: bacterial microcrystalline cellulose; EG: endo- $\beta$-1,4-glucanase; BG: $\beta$-glucosidase.

\section{Authors' contributions}

SYD designed the study and guided the experiment; BS and BL performed the experiments, analyzed data, and drafted the manuscript equally. XW, WS, SP, $A F$, and CC processed, analyzed the data, and modify the manuscript. JDW and SJS reviewed the data and helped write the manuscript. All authors read and approved the final manuscript.

\section{Author details \\ 1 Department of Plant Biology, Michigan State University, East Lansing, Ml 48824, USA. ${ }^{2}$ MSU-DOE Plant Research Laboratory, Michigan State Univer- sity, East Lansing, MI 48824, USA. ${ }^{3}$ DOE Great Lakes Bioenergy Research Center, Michigan State University, East Lansing, MI 48824, USA. ${ }^{4}$ Nanoscience and Nanoengineering Program, South Dakota School of Mines and Technol- ogy, Rapid City, SD 57701, USA.}

\section{Acknowledgements}

We would like to thank Dr. Daniel Jones and Dr. Tony Schilmiller, MSU Mass Spectrometry Facility, for their help with the MS experimental design.

\section{Competing interests}

The authors declare that they have no competing interests.

\section{Availability of data and materials}

The data sets generated and/or analyzed during the present study are included in this published article and are available from BS and SD on reasonable request.

\section{Ethics approval and consent to participate}

Not applicable.

\section{Funding}

This work was funded by the US National Science Foundation (DMRBMART-1206908), the U.S. Department of Energy Great Lakes Bioenergy Research Center (DOE Office of Science BER DE-FC02-07ER64494) and Office of Biological and Environmental Research (DE-SC0012411). Additional funding was provided by grant DE-FG02-91ER200021 to the MSU-DOE Plant Research Laboratory from the U.S. Department of Energy, Office of Basic Energy Sciences, Division of Chemical Sciences, Geosciences and Biosciences.

\section{Publisher's Note}

Springer Nature remains neutral with regard to jurisdictional claims in published maps and institutional affiliations.

Received: 5 October 2017 Accepted: 11 January 2018

Published online: 15 February 2018

\section{References}

1. Bozell JJ, Petersen GR. Technology development for the production of biobased products from biorefinery carbohydrates-the US Department of Energy's "Top 10" revisited. Green Chem. 2010;12:539-54.

2. Himmel ME, Ding SY, Johnson DK, Adney WS, Nimlos MR, et al. Biomass recalcitrance: engineering plants and enzymes for biofuels production. Science. 2007:315:804-7.

3. Kumar R, Singh S, Singh OV. Bioconversion of lignocellulosic biomass: biochemical and molecular perspectives. J Ind Microbiol Biotechnol. 2008;35:377-91.

4. Horn SJ, Vaaje-Kolstad G, Westereng B, Eijsink VGH. Novel enzymes for the degradation of cellulose. Biotechnol Biofuels. 2012;5:45.

5. Ragauskas AJ, Williams CK, Davison BH, Britovsek G, Cairney J, et al. The path forward for biofuels and biomaterials. Science. 2006;311:484-9.

6. Mansfield SD, Mooney C, Saddler JN. Substrate and enzyme characteristics that limit cellulose hydrolysis. Biotechnol Progr. 1999;15:804-16.

7. Lynd LR, Weimer PJ, van ZyI WH, Pretorius IS. Microbial cellulose utilization: fundamentals and biotechnology. Microbiol Mol Biol Rev. 2002;66:506-77.

8. Harris PV, Welner D, MCFarland KC, Re E, Poulsen JCN, et al. Stimulation of lignocellulosic biomass hydrolysis by proteins of glycoside hydrolase family 61: structure and function of a large, enigmatic family. Biochemistry. 2010;49:3305-16.

9. Hu JG, Arantes V, Pribowo A, Gourlay K, Saddler JN. Substrate factors that influence the synergistic interaction of AA9 and cellulases during the enzymatic hydrolysis of biomass. Energ Environ Sci. 2014;7:2308-15.

10. Phillips CM, Beeson WT, Cate JH, Marletta MA. Cellobiose dehydrogenase and a copper-dependent polysaccharide monooxygenase potentiate cellulose degradation by neurospora crassa. ACS Chem Biol. 2011;6:1399-406

11. Banerjee G, Car S, Scott-Craig JS, Borrusch MS, Walton JD. Rapid optimization of enzyme mixtures for deconstruction of diverse pretreatment/ biomass feedstock combinations. Biotechnol Biofuels. 2010;3:22.

12. Davies G, Henrissat B. Structures and Mechanisms of Glycosyl Hydrolases. Structure. 1995;3:853-9.

13. Kracher D, Scheiblbrandner S, Felice AKG, Breslmayr E, Preims M, et al. Extracellular electron transfer systems fuel cellulose oxidative degradation. Science. 2016;352:1098-101.

14. Quinlan RJ, Sweeney MD, Lo Leggio L, Otten H, Poulsen JCN, et al. Insights into the oxidative degradation of cellulose by a copper metalloenzyme that exploits biomass components. Proc Natl Acad Sci USA. 2011;108:15079-84

15. Vaaje-Kolstad G, Westereng B, Horn SJ, Liu ZL, Zhai H, et al. An oxidative enzyme boosting the enzymatic conversion of recalcitrant polysaccharides. Science. 2010;330:219-22.

16. Westereng B, Ishida T, Vaaje-Kolstad G, Wu M, Eijsink VGH, et al. The putative endoglucanase PCGH61D from phanerochaete chrysosporium is a metal-dependent oxidative enzyme that cleaves cellulose. PLOS ONE. 2011;6. https://doi.org/10.1371/journal.pone.0027807.

17. Westereng B, Cannella D, Agger JW, Jorgensen H, Andersen ML, et al. Enzymatic cellulose oxidation is linked to lignin by long-range electron transfer. Sci Rep. 2015;5:18561.

18. Dufrene YF, Ando T, Garcia R, Alsteens D, Martinez-Martin D, et al. Imaging modes of atomic force microscopy for application in molecular and cell biology. Nat Nanotechnol. 2017;12:295-307.

19. Villares A, Moreau C, Bennati-Granier C, Garajova S, Foucat L, et al. Lytic polysaccharide monooxygenases disrupt the cellulose fibers structure. Sci Rep. 2017;7:40262. 
20. Teeri TT. Crystalline cellulose degradation: new insight into the function of cellobiohydrolases. Trends Biotechnol. 1997;15:160-7.

21. Hemsworth GR, Johnston EM, Davies GJ, Walton PH. Lytic polysaccharide monooxygenases in biomass conversion. Trends Biotechnol. 2015;33:747-61.

22. Beeson WT, Vu VV, Span EA, Phillips CM, Marletta MA. Cellulose degradation by polysaccharide monooxygenases. Annu Rev Biochem. 2015;84:923-46

23. Isaksen T, Westereng B, Aachmann FL, Agger JW, Kracher D, et al. A C4-oxidizing lytic polysaccharide monooxygenase cleaving both cellulose and cello-oligosaccharides. J Biol Chem. 2014;289:2632-42.

24. Chen CR, Makhatadze Gl. ProteinVolume: calculating molecular van der Waals and void volumes in proteins. BMC Bioinformatics. 2015;16:101.

25. Igarashi K, Uchihashi T, Koivula A, Wada M, Kimura S, et al. Traffic jams reduce hydrolytic efficiency of cellulase on cellulose surface. Science. 2011;333:1279-82.

26. Liu YS, Baker JO, Zeng YN, Himmel ME, Haas T, et al. Cellobiohydrolase hydrolyzes crystalline cellulose on hydrophobic faces. J Biol Chem. 2011;286:11195-201.

27. Divne C, Stahlberg J, Teeri TT, Jones TA. High-resolution crystal structures reveal how a cellulose chain is bound in the 50 angstrom long tunnel of cellobiohydrolase I from Trichoderma reesei. J Mol Biol. 1998;275:309-25.

28. Karkehabadi S, Hansson H, Kim S, Piens K, Mitchinson C, et al. The first structure of a glycoside hydrolase family 61 member, Cel61B from Hypocrea jecorina, at 1.6 angstrom resolution. J Mol Biol. 2008;383:144-54.

29. Martinez-Sanz M, Lopez-Sanchez P, Gidley MJ, Gilbert EP. Evidence for differential interaction mechanism of plant cell wall matrix polysaccharides in hierarchically-structured bacterial cellulose. Cellulose. 2015;22:1541-63.

30. Martinez-Sanz M, Gidley MJ, Gilbert EP. Hierarchical architecture of bacterial cellulose and composite plant cell wall polysaccharide hydrogels using small angle neutron scattering. Soft Matter. 2016;12:1534-49.

31. White AR, Brown RM. Enzymatic-hydrolysis of cellulose-visual characterization of the process. Proc Natl Acad Sci USA. 1981;78:1047-51.

32. Kim IJ, Youn HJ, Kim KH. Synergism of an auxiliary activity 9 (AA9) from Chaetomium globosum with xylanase on the hydrolysis of xylan and lignocellulose. Process Biochem. 2016;51:1445-51.

33. Banerjee G, Car S, Scott-Craig JS, Borrusch MS, Aslam N, et al. Synthetic enzyme mixtures for biomass deconstruction: production and optimization of a core set. Biotechnol Bioeng. 2010;106:707-20.
34. Krystynowicz A, Czaja W, Wiktorowska-Jezierska A, Goncalves-Miskiewicz $M$, Turkiewicz $M$, et al. Factors affecting the yield and properties of bacterial cellulose. J Ind Microbiol Biotechnol. 2002;29:189-95.

35. McKenna BA, Mikkelsen D, Wehr JB, Gidley MJ, Menzies NW. Mechanical and structural properties of native and alkali-treated bacterial cellulose produced by Gluconacetobacter xylinus strain ATCC 53524. Cellulose. 2009;16:1047-55.

36. Yang B, Willies DM, Wyman CE. Changes in the enzymatic hydrolysis rate of avicel cellulose with conversion. Biotechnol Bioeng. 2006:94:1122-8.

37. Agger JW, Isaksen T, Varnai A, Vidal-Melgosa S, Willats WGT, et al. Discovery of LPMO activity on hemicelluloses shows the importance of oxidative processes in plant cell wall degradation. P Natl Acad Sci USA. 2014;111:6287-92.

38. Forsberg Z, Vaaje-Kolstad G, Westereng B, Bunaes AC, Stenstrom Y, et al. Cleavage of cellulose by a CBM33 protein. Protein Sci. 2011;20:1479-83.

39. Li X, Beeson WT, Phillips CM, Marletta MA, Cate JHD. Structural basis for substrate targeting and catalysis by fungal polysaccharide monooxygenases. Structure. 2012;20:1051-61.

40. Beeson WT, Phillips CM, Cate JHD, Marletta MA. Oxidative cleavage of cellulose by fungal copper-dependent polysaccharide monooxygenases. J Am Chem Soc. 2012;134:890-2.

41. Bennati-Granier C, Garajova S, Champion C, Grisel S, Haon M, et al. Substrate specificity and regioselectivity of fungal AA9 lytic polysaccharide monooxygenases secreted by Podospora anserina. Biotechnol Biofuels. 2015;8.

42. Frommhagen M, Koetsier MJ, Westphal AH, Visser J, Hinz SWA, et al. Lytic polysaccharide monooxygenases from Myceliophthora thermophila C1 differ in substrate preference and reducing agent specificity. Biotechnol Biofuels. 2016;9.

43. Vu W, Beeson WT, Phillips CM, Cate JHD, Marletta MA. Determinants of regioselective hydroxylation in the fungal polysaccharide monooxygenases. J Am Chem Soc. 2014;136:562-5.

44. Bey M, Zhou SM, Poidevin L, Henrissat B, Coutinho PM, et al. Cello-oligosaccharide oxidation reveals differences between two lytic polysaccharide monooxygenases (Family GH61) from podospora anserina. Appl Environ Microb. 2013;79:488-96.

\section{Submit your next manuscript to BioMed Central and we will help you at every step:}

- We accept pre-submission inquiries

- Our selector tool helps you to find the most relevant journal

- We provide round the clock customer support

- Convenient online submission

- Thorough peer review

- Inclusion in PubMed and all major indexing services

- Maximum visibility for your research

Submit your manuscript at www.biomedcentral.com/submit
() Biomed Central 